\title{
A case of tetanus infection following hand injury.
}

\author{
Nitsan Z, Kucuk N, Zariki M, Appel S*, Tichmanovich N, Gefel D, Kagarel R, Shaviv E, Maayan S, Milo R \\ Barzilai Medical Center, Ashkelon, Israel
}

\begin{abstract}
Tetanus is a disease caused by a neurotoxin produced by the Gram-positive bacillus Clostridium tetani $(C$. tetani), found in the soil and human and animal feces. $C$. tetani is a mobile, spore-forming obligate anaerobe. Under anaerobic conditions as in necrotic and infected tissue, the tetanus bacillus secretes two toxins: tetanospasmin and tetanolysin. Tetanospasmin leads to the clinical syndrome of tetanus comprised of a triad of rigidity, muscle spasms and, if severe, autonomic dysfunction. We describe a case of a 31-year-old patient who presented with painful stiffness especially of the trunk, intermittent diffuse muscle spasms, difficulty swallowing and opening his mouth. After clinical workup a diagnosis of tetanus was made. The patient was treated with active vaccination by tetanus toxoid (TDaP), and passive vaccination (Tetanus Immune Globulin -TIG), antibiotics, pain relief agents and muscle relaxants and his symptoms resolved gradually. He was discharged from the hospital in a good condition 14 day after his admission.
\end{abstract}

Keywords: Tetanus, Necrotic, Autonomic dysfunction, Endotracheal intubation, C. tetani.

\section{Introduction}

Tetanus is caused by an infection with the bacterium Clostridium tetani [1] which is commonly found in soil, saliva, dust, and manure [2]. Tetanus is a disease that leads to muscle contractions that stay that way for some time. Infection generally occurs through wound contamination, and often involves a cut or deep puncture woundThe bacteria generally enter through a break in the skin such as a cut or puncture wound by a contaminated object [2]. They produce toxins that interfere with muscle contractions, resulting in the typical signs [3]. Diagnosis is based on the presenting signs and symptoms [1]. Tetanus occurs in all parts of the world but is most frequent in hot and wet climates where the soil contains a lot of organic matter.

\section{Case Report}

A 31-year-old Sudanese male sanitation worker in a municipality, was referred to the emergency department with complaints of difficulty in opening his mouth and swallowing and painful stiffness in his back and chest that started three days prior to his admission. Symptoms were aggravated by breathing and movement. The general stiffness in his body increased gradually. The spasms of the back muscles were compatible with opisthotonos. The patient had no known medical history, did not take any medications and was unvaccinated.

Vital signs were significant for mild hypertension temperature was 36 centigrade, his initial blood pressure measurement was $140 / 100 \mathrm{mmHg}$, pulse was $76 / \mathrm{min}$ but a few hours later blood pressure was $160 / 100 \mathrm{mmHg}$ and pulse were $87 / \mathrm{min}$ with no change in the other vital signs. The oxygen saturation was stable around $98 \%$. His BMI was calculated as 17 .

Laboratory results were non-significant and showed white blood cell count of $9.820 \mathrm{~K} / \mathrm{micro} \mathrm{L}$ and decreased gradually from then to the middle of normal range. Toxicology tests and routine blood and urine tests were normal.
The patient was fully oriented to time, place and situation. Physical examination was significant for an unhealed wound on his right hand that was caused by a metal cut approximately a month prior to his admission. The wound ( $3 \mathrm{~cm}$ in diameter) was in a process of forming a scab in the back of the right hand (Figure 1). Neurological examination showed neck stiffness, intermittent lockjaw, dysphagia, extreme stiffness in trunk and limbs with intermittent diffuse muscle spasms. Plantar responses were bilaterally extensor (positive Babinski sign). Stimulation of the posterior pharyngeal wall (spatula test) resulted in biting the triggering tongue depressor (craft stick) due to spasm of the masseter muscles. Due to initial presentation of chest pain, a

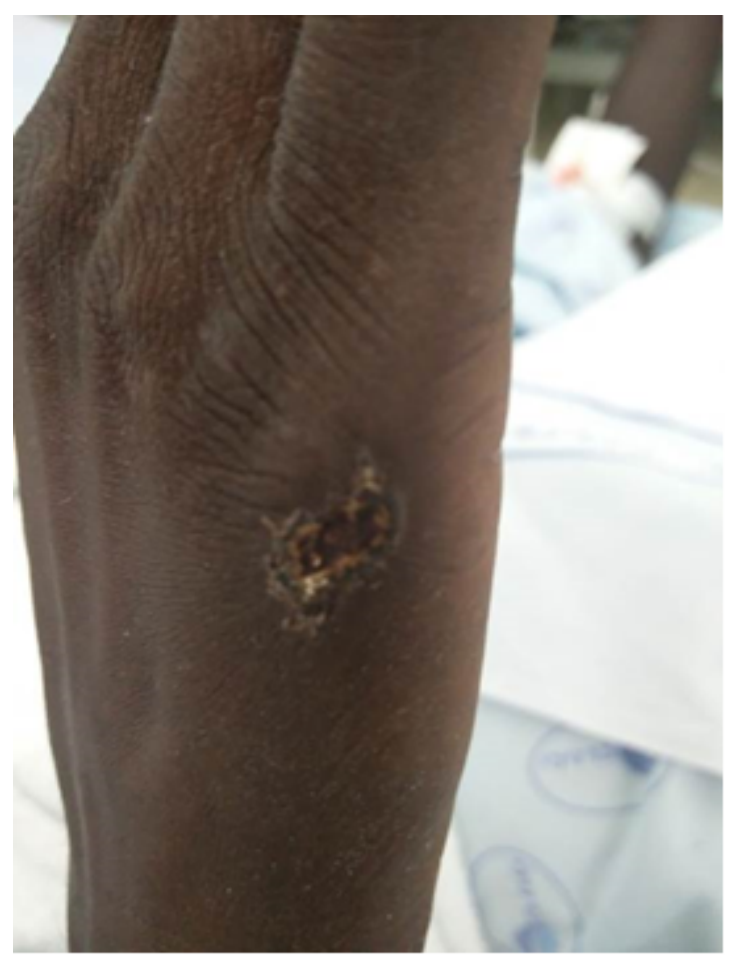

Figure 1. The wound in the right hand of the patient. 
basic cardiological workup was performed. Echocardiography was within normal limits ruling out pericarditis and myocardial infarction.

A working diagnosis of tetanus infection was performed, and the patient received at first a passive vaccination (Tetanus Immune Globulin, TIG) and an active vaccination (tetanus toxoid, TDaP) in the same arm in close proximity, with accordance with an infectious disease consultant recommendation. Due to the fact that those two types of vaccinations could contradict the effect of each other, a second passive vaccination was made in the other arm (making a totally of two passive vaccinations and one active vaccination)

He was treated with Tetanus Immune Globulin (TIG), in a dose of 3000 units intramuscularly and with tetanus toxoid (TDap), $0.5 \mathrm{ml}$ intramuscularly. He was also treated with Antibiotic, Penicillin, Flagil and Metronidazole intravenously was also administered. To relieve his painful spasms, he was given Diclofenac $75 \mathrm{mg}$ intramuscularly and benzodiazepines. His vital signs were monitored continuously.

The treatment team considered an option of prophylactic endotracheal intubation against the possibility of deterioration in patient's condition and necessity for artificial respiration, but the rapid improvement in his state made it unnecessary. A general surgery consultant examined the wound of the cut in the right hand and a biopsy of tissue from the wound was taken for culture, but there was no growing of the Clostridium tetani bacilli.

Anti-glutamic acid decarboxylase (anti-GAD) antibody titers in the serum were measured within the normal range at $1 \mathrm{U} /$ $\mathrm{mL}$. On the fourth day of his hospitalization his condition showed marked improvement. He was transferred from ICU to general medicine department for observation and follow-up and discharged from the hospital in good condition 14 days since his hospitalization.

\section{Discussion}

Tetanus is caused by a neurotoxin produced by the Gram-positive bacillus Clostridium tetani (C. tetani) which is found in the soil and human and animal faeces. $C$. tetani is a mobile, sporeforming obligate anaerobe [1]. Under anaerobic conditions as in necrotic and infected tissue, the tetanus bacillus secretes two toxins: tetanospasmin and tetanolysin. Tetanospasmin leads to the clinical syndrome of tetanus [2]. There is a clinical triad of rigidity, muscle spasms and, if severe, autonomic dysfunction [3]. Because of the extreme potency of the toxin, tetanus disease does not result in tetanus immunity. Active immunization with tetanus toxoid should begin or continue as soon as the person's condition has stabilized [4]. The major differential diagnosis included dystonic reaction to a drug, but the patient denied any drug treatment or use and there was no evidence for abnormal finding in the urine and blood tests for toxicology (including strychnine poisoning). Malignant hyperthermia (MH) and neuroleptic malignant syndrome (NMS) were not likely because there was no fever, the CPK level was 700 (less then usually expected in $\mathrm{MH}$ and NMS) and there was no evidence of neuroleptic drug use. Option of insect bite with reaction to its toxin (like black widow spider biting) also was not likely because the patient denied any episode of sharp pain, he did not see any unusual insect in his surroundings and there was no any sign of biting in his physical examination. The likelihood of "stiff person" syndrome was not been supported by the normal result of the anti-GAD antibodies. The elevation in blood pressure and pulse was most probably due to his pain but could also represent early autonomic instability due to the tetanus infection. In tetanus, the incubation period (time from injury to first symptom) is about 7-10 days. The period of onset is the time from the first symptom to the first spasm and varies from 1 to 7 days. A short incubation period and period of onset are associated with more severe disease [5]. A time interval of less than 7 days between the exposure to the tetanus and the clinical manifestation of the infection is commonly considered as a bad prognostic factor, on the contrary, time interval of more than one week is commonly considered as a good prognostic factor [6].

\section{Conclusion}

In our case, the relatively prolonged latency, which was more than 3 weeks, between the exposure to $C$. tetani and the appearance of the clinical symptoms can explain the relatively quick recovery of the patient.

\section{References}

1. Schwartz E, Rodeisperger E. Skin and soft tissue infections. In: Schillinger D, Harwood-Nuss A (eds.) Infections in Emergency Medicine. 1990;2:63-113.

2. Parker J. Managing tetanus. Emerg Nurse. 2006;14:14-7.

3. Alfery DD, Rauscher A. Tetanus: A review. Crit Care Med. 1979;4:176-81.

4. Gibson K, Uwineza JB, Kiviri W, et al. Tetanus in developing countries: A case series and review. Can J Anaesth. 2009;56:307-15.

5. Phillips LA. A classification of tetanus. Lancet. 1967;1:1216-17.

6. Saltoglu N, Tasova Y, Midikli D, et al. Prognostic factors affecting deaths from adult tetanus. Clin Microbiol Infect. 2004;10:229-33.

\section{*Correspondence to:}

\section{Appel S}

Barzilai Medical Center

Ashkelon

Israel

Tel: +972 526767130

E-mail: shmuela@barzi.health.gov.il 